\title{
Chirality-Induced Spin Selectivity in a Coarse-Grained Tight-Binding Model for Helicene
}

Matthias Geyer, ${ }^{*, \dagger, \dagger}$ Rafael Gutierrez, ${ }^{*, \dagger}$ Vladimiro Mujica,,${ }^{\mathbf{S}} \S$ and Gianaurelio Cuniberti ${ }^{\dagger}, \|, \ddagger$

$\dagger$ Institute for Materials Science and Max Bergmann Center of Biomaterials, TU Dresden, 01062 Dresden, Germany

$\ddagger$ Dresden Center for Computational Materials Science (DCMS), TU Dresden, 01062 Dresden, Germany

ๆSchool of Molecular Sciences, Arizona State University, Tempe, Arizona, 85287, USA

$\S$ Donostia International Physics Center (DIPC), P.K. 1072, 20080 Donostia, Euskadi, Spain

\|Center for Advancing Electronics Dresden, TU Dresden, 01062 Dresden, Germany

E-mail: matthias.geyer@tu-dresden.de; rafael.gutierrez@tu-dresden.de 


\section{Supplementary Information}

\section{The decimation procedure}

Generally speaking, the decimation procedure used here aims for simplification of a system by reducing the dimension of its state space $\left(\mathrm{se}^{\sqrt{12}}\right)$. This shall be illustrated for the following system:

Consider a Hamiltonian

$$
H=\left(\begin{array}{ccc}
\varepsilon_{1} & t & r \\
t & \varepsilon_{2} & s \\
r & s & \varepsilon_{3}
\end{array}\right)
$$

with all entries being real. The eigenvalue equation is $\varepsilon \psi=H \psi$ or explicitly (with $\psi=$ $\left.\left(u_{1}, u_{2}, u_{3}\right)\right)$

$$
\begin{aligned}
& \varepsilon u_{1}=\varepsilon_{1} u_{1}+t u_{2}+r u_{3} \\
& \varepsilon u_{2}=\varepsilon_{2} u_{2}+t u_{1}+s u_{3} \\
& \varepsilon u_{3}=\varepsilon_{3} u_{3}+r u_{1}+s u_{2} .
\end{aligned}
$$

If one wants to eliminate state 2 one solves the second equation in (2) with $\varepsilon$ replaced by $\varepsilon_{\mathrm{c}}$ for $u_{2}$ and plugs the result

$$
u_{2}=\frac{t u_{1}+s u_{3}}{\varepsilon_{\mathrm{c}}-\varepsilon_{2}}
$$

into the first and second equation. In general one has to solve a system of equations for the states one wants to eliminate. This yields the effective eigenvalue equations

$$
\begin{aligned}
& \left(\varepsilon-\varepsilon_{1}-\frac{t^{2}}{\varepsilon_{\mathrm{c}}-\varepsilon_{2}}\right) u_{1}=\left(r+\frac{t s}{\varepsilon_{\mathrm{c}}-\varepsilon_{2}}\right) u_{3} \\
& \left(\varepsilon-\varepsilon_{3}-\frac{s^{2}}{\varepsilon_{\mathrm{c}}-\varepsilon_{2}}\right) u_{3}=\left(r+\frac{t s}{\varepsilon_{\mathrm{c}}-\varepsilon_{2}}\right) u_{1}
\end{aligned}
$$


and thus an effective Hamiltonian

$$
H_{\mathrm{eff}}\left(\varepsilon_{\mathrm{c}}\right)=\left(\begin{array}{cc}
\varepsilon_{1}+\frac{t^{2}}{\varepsilon_{\mathrm{c}}-\varepsilon_{2}} & r+\frac{t s}{\varepsilon_{\mathrm{c}}-\varepsilon_{2}} \\
r+\frac{t s}{\varepsilon_{\mathrm{c}}-\varepsilon_{2}} & \varepsilon_{3}+\frac{s^{2}}{\varepsilon_{\mathrm{c}}-\varepsilon_{2}}
\end{array}\right)
$$

Note that the equation

$$
\operatorname{det}\left(\varepsilon \mathbf{1}-H_{\mathrm{eff}}(\varepsilon)\right)=0
$$

has as roots the eigenvalues of the full Hamiltonian. Also through the procedure, an effective coupling between the states 1 and 3 arises which contains a correction to the initial coupling $r$. This correction can be interpreted as the result of the coupling chain

$$
1 \stackrel{t}{\longrightarrow} 2 \stackrel{t}{\longrightarrow} 3
$$

The choice of the parameter $\varepsilon_{\mathrm{c}}$ then ensures that the new system is an approximation of the old one.

If one is only interested in the effective couplings one can omit e.g. the $u_{1}$ contribution to $u_{2}$ in Eq. (3) before plugging it into the first equation in (2) and so on. This way one does not get eigenenergy corrections but, in systems with a larger state space, also avoids higher order contributions in electronic couplings and greatly reduces the calculation effort.

In the present example, this can be seen as follows:

Let for simplicity $r=0, \varepsilon_{1}=\varepsilon_{3} \neq \varepsilon_{2}$ and $t^{2}$ and $s^{2}$ be small compared to $\left|\varepsilon_{1}-\varepsilon_{2}\right|$. The eigenvalues of $H$ are then $\varepsilon_{1}$ and $\frac{1}{2}\left( \pm \sqrt{4\left(s^{2}+t^{2}\right)+\left(\varepsilon_{1}-\varepsilon_{2}\right)^{2}}+\varepsilon_{1}+\varepsilon_{2}\right)$ which are obviously also the solutions of Eq. (6).

The unperturbed system is described by the Hamiltonian diagonal $H_{0}=\left(\begin{array}{ccc}\varepsilon_{1} & 0 & 0 \\ 0 & \varepsilon_{2} & 0 \\ 0 & 0 & \varepsilon_{3}\end{array}\right)$ 
with obvious eigenvalues and eigenvectors. The perturbation is $V=\left(\begin{array}{ccc}0 & t & 0 \\ t & 0 & s \\ 0 & s & 0\end{array}\right)$. Let $|n\rangle$ be the eigenvectors of $H_{0}$, then the first order corrections in Rayleigh-Schrödinger perturbation theory to the eigenenergies $\varepsilon_{1}$ and $\varepsilon_{3}$ vanish because $\langle n|V| n\rangle=0$ for $n=1,3$. However the second order corrections are

$$
\varepsilon_{n}^{(2)}=\sum_{k \neq n} \frac{|\langle k|V| n\rangle|^{2}}{\varepsilon_{n}-\varepsilon_{k}}
$$

which yields exactly the energy corrections from Eq. (4) with $\varepsilon_{\mathrm{c}}=\varepsilon_{n}$ for $n=1,3$.

To move beyond this simple example, we employ Löwdin partitioning as described e.g. in ${ }^{3}$. Let $P$ be an orthogonal projector on the system's state space, i. e. $P^{2}=P$ and $P^{\dagger}=P$. $P$ and $P^{\perp}=\mathrm{id}-P$ are then associated with mutually orthogonal subspaces of the state space. We now want to eliminate all states of the form $P^{\perp}|\psi\rangle$ to obtain an effective Hamiltonian acting only on the $P|\psi\rangle$ states. To this end consider the eigenvalue equation

$$
H|\psi\rangle=\varepsilon|\psi\rangle
$$

and apply $P$ and $P^{\perp}$ to both sides to get

$$
P H P|\psi\rangle+P H P^{\perp}|\psi\rangle=\varepsilon P|\psi\rangle
$$

and

$$
P^{\perp} H P|\psi\rangle+P^{\perp} H P^{\perp}|\psi\rangle=\varepsilon P^{\perp}|\psi\rangle
$$

If $\varepsilon-P^{\perp} H P^{\perp}$ is invertible one can rewrite Eq. (11) to get

$$
P^{\perp}|\psi\rangle=\left(\varepsilon-P^{\perp} H P^{\perp}\right)^{-1} P^{\perp} H P|\psi\rangle
$$


and thus by plugging 12 into 10$)$ and using $P^{\perp}=\left(P^{\perp}\right)^{2}$

$$
\underbrace{\left(P H P+P H P^{\perp}\left(\varepsilon-P^{\perp} H P^{\perp}\right)^{-1} P^{\perp} H P\right)}_{=H_{\mathrm{eff}}}|\psi\rangle=\varepsilon P|\psi\rangle
$$

which is an eigenvalue equation for the effective Hamiltonian $H_{\text {eff }}$ with eigenstates of the form $P|\psi\rangle$. In other words if $\varepsilon$ is an eigenvalue of $H$ with eigenstate $|\psi\rangle$ then it is also an eigenvalue of $H_{\text {eff }}$ with eigenstate $P|\psi\rangle$.

Now let $P$ be the projection onto the eigenspace of $H$ to eigenvalue $\varepsilon_{0}$ of some Hamiltonian $H_{0}$ with

$$
H=H_{0}+H_{1} \text {. }
$$

Expanding $\left(\varepsilon-P^{\perp} H P^{\perp}\right)^{-1}$ into a power series yields

$$
\begin{aligned}
\left(\varepsilon-P^{\perp} H P^{\perp}\right)^{-1} & =\left(\varepsilon_{0}-P^{\perp} H_{0} P^{\perp}\right)^{-1} \sum_{n=0}^{\infty}\left(P^{\perp} H_{1} P^{\perp}+\varepsilon_{0}-\varepsilon\right)^{n}\left(\varepsilon_{0}-P^{\perp} H_{0} P^{\perp}\right)^{-n} \\
& \approx\left(\varepsilon_{0}-P^{\perp} H_{0} P^{\perp}\right)^{-1}
\end{aligned}
$$

So we get

$$
H_{\mathrm{eff}} \approx \varepsilon_{0} P+P H_{1} P+P H_{1} P^{\perp}\left(\varepsilon_{0}-P^{\perp} H_{0} P^{\perp}\right)^{-1} P^{\perp} H_{1} P .
$$

We shall give an example how to calculate $H_{\text {eff }}$ in our model in the following section.

\section{Geometry and microscopic couplings of the helicene model}

The set of sites was divided into three subsets $\mathcal{I}$ (the inner helix) as well as $\mathcal{M}$ and $\mathcal{A}$ which consist of all particles on the outer helix. The sites are enumerated from 1 to 26. The sites 1 to 19 are in $\mathcal{M}$ and $\mathcal{A}$ and the sites from 20 to 26 are contained in $\mathcal{I}$. Explicitly one defines 
these sets as follows:

$$
\begin{aligned}
\mathcal{I} & :=\{n \in \mathbb{N}\} 20 \leq n \leq 26 \\
\mathcal{M} & :=\{n \in \mathbb{N}\} \exists k \in \mathbb{N}_{0}: n=3 k+1 \wedge 0 \leq k \leq 6 \\
\mathcal{A} & :=\{n \in \mathbb{N}\} 1 \leq n \leq 19 \backslash \mathcal{M} .
\end{aligned}
$$

Note that $n \in \mathcal{M} \Leftrightarrow n \in\{1, \ldots, 19\} \wedge(n-1) \bmod 3=0$.

One can also define the neighbour relations

$$
\begin{aligned}
& \mathcal{N}_{\mathcal{I}}:=\{(n, m) \in \mathcal{I} \times \mathcal{I}\}|n-m|=1 \text { and } \\
& \mathcal{N}_{\mathcal{A}}:=\{(n, m) \in \mathcal{A} \times \mathcal{A}\}|n-m|=1
\end{aligned}
$$

as well as

$$
\begin{aligned}
& \mathcal{N}_{(\mathcal{I}, \mathcal{M})}:=\{(n, m) \in \mathcal{I} \times \mathcal{M} \cup \mathcal{M} \times \mathcal{I}\} \frac{n-1}{3}=m-20 \vee \frac{m-1}{3}=n-20, \\
& \mathcal{N}_{(\mathcal{M}, \mathcal{A})}:=\{(n, m) \in \mathcal{M} \times \mathcal{A}\} n-m=-1 \cup\{(n, m) \in \mathcal{A} \times \mathcal{M}\} n-m=1 \text { and } \\
& \mathcal{N}_{(\mathcal{A}, \mathcal{M})}:=\{(n, m) \in \mathcal{M} \times \mathcal{A}\} n-m=1 \cup\{(n, m) \in \mathcal{A} \times \mathcal{M}\} n-m=-1 .
\end{aligned}
$$

Now the site-dependence of $R_{i}$ and $\Phi_{i}$ in

$$
\mathbf{R}_{i}=\left(R_{i} \cos \Phi_{i}, R_{i} \sin \Phi_{i}, \frac{b}{2 \pi} \Phi_{i}\right)
$$

can be given:

$$
R_{i}= \begin{cases}r_{0} & \text { for } i \in \mathcal{N} \\ r_{1} & \text { for } i \in \mathcal{M} \\ r_{2} & \text { for } i \in \mathcal{A}\end{cases}
$$

and

$$
\Phi_{i}=\left\{\begin{array}{ll}
(i-1) \phi_{0} & \text { for } i \in \mathcal{N} \\
\left\lfloor\frac{i}{3}\right\rfloor \cdot \phi_{2}+\left(i-1-\left\lfloor\frac{i}{3}\right\rfloor\right) \phi_{1} & \text { for } i \in \mathcal{M} \cup \mathcal{A}
\end{array} .\right.
$$


Only $s^{-}, x_{-}, y$ - and $z$-orbitals are considered at each site. The couplings between these orbitals are given by the formula

$$
H_{\mu \nu}^{(i j)}=\mathbf{n}(\mu, i j)^{\|} \cdot \mathbf{n}(\nu, j i)^{\|} V_{\mu \nu \sigma}+\mathbf{n}(\mu, i j)^{\perp} \cdot \mathbf{n}(\nu, j i)^{\perp} V_{\mu \nu \pi}
$$

with

$$
\mathbf{n}(\mu, i j)^{\|}= \begin{cases}\frac{\mathbf{R}_{j i} \cdot \mathbf{n}(\mu, i)}{\left\|\mathbf{R}_{j i}\right\|^{2}} \mathbf{R}_{j i} & \text { for } \mu \in\{x, y, z\} \\ \frac{\mathbf{R}_{j i}}{\left\|\mathbf{R}_{j i}\right\|} & \text { for } \mu=s\end{cases}
$$

and

$$
\mathbf{n}(\mu, i j)^{\perp}=\left\{\begin{array}{ll}
\mathbf{n}(\mu, i)-\frac{\mathbf{R}_{j i} \cdot \mathbf{n}(\mu, i)}{\left\|\mathbf{R}_{j i}\right\|^{2}} \mathbf{R}_{j i} & \text { for } \mu \in\{x, y, z\} \\
0 & \text { for } \mu=s
\end{array} .\right.
$$

Here $\mathbf{R}_{i j}=\mathbf{R}_{i}-\mathbf{R}_{j}$ and $\mathbf{n}(\mu, i)$ are the local directions of the orbital axes given by $\mathbf{n}(x, i)=$ $\left(\cos \Phi_{i}, \sin \Phi_{i}, 0\right), \mathbf{n}(y, i)=\left(-\sin \Phi_{i}, \cos \Phi_{i}, 0\right)$ and $\mathbf{n}(z, i)=(0,0,1)$.

So the Hamiltonian before decimation can be written as

$$
H=\sum_{i} H_{i}+\sum_{i, j} H_{i j}
$$

with

$$
H_{i j}=\sum_{\mu, \nu} H_{\mu \nu}^{(i j)} a_{\mu, i}^{\dagger} a_{\nu, j}
$$

and

$$
H_{i}=\sum_{\mu, \nu} h_{\mu \nu}^{(i)} a_{\mu, i}^{\dagger} a_{\nu, i}
$$

$H_{i}$ contains the spin-orbit and external field interactions which are short range and thus 
couple only orbitals at the same site with

$$
h^{(i)}=\left(\begin{array}{cccc}
\varepsilon_{s} & E_{\mathrm{n}} & E_{\mathrm{t}} & E_{z} \\
E_{\mathrm{n}} & \varepsilon_{p \sigma} & -i \sigma_{z} \xi_{p} & i \sigma_{y} \xi_{p} \\
E_{\mathrm{t}} & i \sigma_{z} \xi_{p} & \varepsilon_{p \sigma} & -i \sigma_{x} \xi_{p} \\
E_{z} & -i \sigma_{y} \xi_{p} & i \sigma_{x} \xi_{p} & \varepsilon_{p \pi}
\end{array}\right)
$$

For the decimation, all interactions between different orbitals and sites (i.e. all off-diagonal elements) are treated as perturbations. The effective Hamiltonian shall only act on $z$-orbitals so the projection $P$ is defined by

$$
P|\mu, i\rangle=\delta_{z \mu}|\mu, i\rangle
$$

We shall now treat the example given in the article within the partitioning framework layed out in Eqs. (9) - (16) so we again neglect the SOC and set $E_{\mathrm{n}}=E_{\mathrm{t}}=0$. We obtain

$$
\begin{aligned}
& \left(P^{\perp}\left(\varepsilon_{0}-P^{\perp} H_{0} P^{\perp}\right)^{-1} P^{\perp}\right)_{i j}= \\
& \left(\begin{array}{ccc}
\left(\varepsilon_{p \pi}-\varepsilon_{s}\right)^{-1} & 0 & 0 \\
0 & \left(\varepsilon_{p \pi}-\varepsilon_{p \sigma}\right)^{-1} & 0 \\
0 & 0 & \left(\varepsilon_{p \pi}-\varepsilon_{p \sigma}\right)^{-1}
\end{array}\right) \delta_{i j}
\end{aligned}
$$

and Eq. 16 turns into

$$
H_{\mathrm{eff}}=\varepsilon_{p \pi} P+P H_{1} P+P H_{1} P^{\perp}\left(\varepsilon_{0}-P^{\perp} H_{0} P^{\perp}\right)^{-1} P^{\perp} H_{1} P .
$$

Also

$$
\left(P H_{1} P\right)_{\mu, i ; \nu, j}=\delta_{\mu z} \delta_{\nu z} H_{z z}^{(i j)}
$$


and

$$
\begin{aligned}
& \left(P H_{1} P^{\perp}\right)_{\mu, i ; \nu, j}=\delta_{\mu z}\left(h_{z \nu}^{(i)} \delta_{i j}+H_{z \nu}^{(i j)}\right) \\
& \left(P^{\perp} H_{1} P\right)_{\mu, i ; \nu, j}=\delta_{\mu z}\left(h_{\nu z}^{(i)} \delta_{i j}+H_{\nu z}^{(i j)}\right)
\end{aligned}
$$

for $\nu=s, x, y$ and zero for $\nu=z$ holds and thus

$$
\begin{aligned}
H_{\mathrm{eff}} & =\varepsilon_{p \pi} \sum_{i} a_{z, i}^{\dagger} a_{z, i}+\sum_{(i, j)} H_{z z}^{(i j)} a_{z, i}^{\dagger} a_{z, j}+\sum_{i} \frac{E_{z}^{2}}{\varepsilon_{p \pi}-\varepsilon_{s}} a_{z, i}^{\dagger} a_{z, i} \\
& +\sum_{i, j} \frac{E_{z} H_{s z}^{(i j)}}{\varepsilon_{p \pi}-\varepsilon_{s}} a_{z, i}^{\dagger} a_{z, j}+\sum_{i, j} \frac{E_{z} H_{z s}^{(i j)}}{\varepsilon_{p \pi}-\varepsilon_{s}} a_{z, i}^{\dagger} a_{z, j} \\
& +\sum_{i, k} \frac{H_{z s}^{(i j)} H_{s z}^{(j k)}}{\varepsilon_{p \pi}-\varepsilon_{s}} a_{z, i}^{\dagger} a_{z, k}+\sum_{i, k} \sum_{\nu=x, y} \frac{H_{z \nu}^{(i j)} H_{\nu z}^{(j k)}}{\varepsilon_{p \pi}-\varepsilon_{p \sigma}} a_{z, i}^{\dagger} a_{z, k}
\end{aligned}
$$

which can be rearranged into

$$
\begin{aligned}
H_{\mathrm{eff}} & =\sum_{i}\left(\varepsilon_{p \pi}+\frac{E_{z}^{2}}{\varepsilon_{p \pi}-\varepsilon_{s}}+\sum_{j} \frac{H_{z s}^{(i j)} H_{s z}^{(j i)}}{\varepsilon_{p \pi}-\varepsilon_{s}}\right) a_{z, i}^{\dagger} a_{z, i} \\
& +\sum_{(i, j)} H_{z z}^{(i j)} a_{z, i}^{\dagger} a_{z, j}+\sum_{(i, j)} \frac{E_{z}\left(H_{z s}^{(i j)}+H_{s z}^{(i j)}\right)}{\varepsilon_{p \pi}-\varepsilon_{s}} a_{z, i}^{\dagger} a_{z, j} \\
& +\sum_{((i, k))} \frac{H_{z s}^{(i j)} H_{s z}^{(j k)}}{\varepsilon_{p \pi}-\varepsilon_{s}} a_{z, i}^{\dagger} a_{z, k}+\sum_{((i, k))} \sum_{\nu=x, y} \frac{H_{z \nu}^{(i j)} H_{\nu z}^{(j k)}}{\varepsilon_{p \pi}-\varepsilon_{p \sigma}} a_{z, i}^{\dagger} a_{z, k}
\end{aligned}
$$

Here $\sum_{(i j)}$ indicates a sum over nearest neighbors while $\sum_{((i j))}$ runs over next-to nearest neighbors. We also treat $E_{z}$ and the $H_{z \mu}^{(i j)}$ 's as independent small parameters and neglect all terms quadratic in one of them (this also justifies the approximation (15)). So $H_{\text {eff }}$ finally becomes

$$
H_{\mathrm{eff}}=\sum_{i} \varepsilon_{p \pi} a_{z, i}^{\dagger} a_{z, i}+\sum_{(i, j)} H_{z z}^{(i j)} a_{z, i}^{\dagger} a_{z, j}+\sum_{(i, j)} \frac{E_{z}\left(H_{z s}^{(i j)}+H_{s z}^{(i j)}\right)}{\varepsilon_{p \pi}-\varepsilon_{s}} a_{z, i}^{\dagger} a_{z, j} .
$$

This interaction can be visualized by the coupling paths

$$
p_{z, i} \stackrel{V_{p p \pi}}{\longrightarrow} p_{z, j}
$$


and

$$
p_{z, i} \stackrel{E_{z}}{\longrightarrow} s_{i} \stackrel{V_{s p \sigma}}{\longrightarrow} p_{z, j}
$$

Because there are three independent small parameters in our calculation, namely the hoppings, the SO coupling $\xi_{p}$ and the external field components, we have to go beyond (15) to take products of all three of them into account and also to include terms which are quadratic in $\xi_{p}$ and the components of $\boldsymbol{E}$. We then use the approximation

$$
\begin{aligned}
& \left(\varepsilon-P^{\perp} H P^{\perp}\right)^{-1}= \\
& \left(\varepsilon_{0}-P^{\perp} H_{0} P^{\perp}\right)^{-1} \sum_{n=0}^{\infty}\left(P^{\perp} H_{1} P^{\perp}+\varepsilon_{0}-\varepsilon\right)^{n}\left(\varepsilon_{0}-P^{\perp} H_{0} P^{\perp}\right)^{-n} \\
& \approx\left(\varepsilon_{0}-P^{\perp} H_{0} P^{\perp}\right)^{-1} \\
& +\left(\varepsilon_{0}-P^{\perp} H_{0} P^{\perp}\right)^{-1}\left(P^{\perp} H_{1} P^{\perp}+\varepsilon_{0}-\varepsilon\right)\left(\varepsilon_{0}-P^{\perp} H_{0} P^{\perp}\right)^{-1} \\
& +\left(\varepsilon_{0}-P^{\perp} H_{0} P^{\perp}\right)^{-1}\left(P^{\perp} H_{1} P^{\perp}+\varepsilon_{0}-\varepsilon\right)^{2}\left(\varepsilon_{0}-P^{\perp} H_{0} P^{\perp}\right)^{-2} .
\end{aligned}
$$

Here the difference $\varepsilon_{0}-\varepsilon$ also has to be expanded in terms of the small parameters. However in our case where $\left(\varepsilon_{0}-P^{\perp} H_{0} P^{\perp}\right)^{-1}$ is simply diagonal (see $(31)$ ) and

$$
\left(\varepsilon_{0}-P^{\perp} H_{0} P^{\perp}\right)^{-1}\left(\varepsilon_{0}-\varepsilon\right)\left(\varepsilon_{0}-P^{\perp} H_{0} P^{\perp}\right)^{-1}
$$

is just a neglegible correction to $\left(\varepsilon_{0}-P^{\perp} H_{0} P^{\perp}\right)^{-1}$ itself if we assume $\varepsilon_{0}$ to be reasonably close to the eigenenergy $\varepsilon$ of the full Hamiltonian, i. e.

$$
\varepsilon=\varepsilon_{0}+\mathcal{O}\left(\left\|H^{(i j)}\right\|\right)+\mathcal{O}\left(\xi_{p}\right)+\mathcal{O}(|\boldsymbol{E}|)
$$

The argumentation for $\left(\varepsilon_{0}-P^{\perp} H_{0} P^{\perp}\right)^{-1} P^{\perp} H_{1} P^{\perp}\left(\varepsilon_{0}-\varepsilon\right)\left(\varepsilon_{0}-P^{\perp} H_{0} P^{\perp}\right)^{-2}$ and $\left(\varepsilon_{0}-P^{\perp} H_{0} P^{\perp}\right)^{-1}\left(\varepsilon_{0}-\varepsilon\right)^{2}\left(\varepsilon_{0}-P^{\perp} H_{0} P^{\perp}\right)^{-2}$ is similar. 


\section{Effective couplings of the helicene model}

The effective couplings of the helicene model obtained via the decimation method sketched in Sec. 1 are listed in the following part of the appendix.

\section{$3.1 \quad$ Kinetic term}

The kinetic term is

$$
\begin{aligned}
H_{\text {kin }} & =t^{\mathrm{I}} \sum_{(i, j)}^{\mathcal{N}_{\mathcal{I}}} a_{i}^{\dagger} a_{j}+t^{\mathrm{A}} \sum_{(i, j)}^{\mathcal{N}_{\mathcal{A}}} a_{i}^{\dagger} a_{j}+t^{\mathrm{I}, \mathrm{M}} \sum_{(i, j)}^{\mathcal{N}_{(\mathcal{I}, \mathcal{M})}} a_{i}^{\dagger} a_{j}+t^{\mathrm{M}, \mathrm{A}} \sum_{(i, j)}^{\mathcal{N}_{(\mathcal{M}, \mathcal{A})}^{\cup \mathcal{N}_{(\mathcal{A}, \mathcal{M})}}} a_{i}^{\dagger} a_{j} \\
& +\tan \left(\frac{\phi_{1}}{2}\right)\left(\rho_{z}^{\mathrm{M}, \mathrm{A}} \sum_{(i, j)}^{\mathcal{N}_{(\mathcal{M}, \mathcal{A})}} a_{i}^{\dagger} a_{j}+\rho_{z}^{\mathrm{A}, \mathrm{M}} \sum_{(i, j)}^{\mathcal{N}_{(\mathcal{A}, \mathcal{M})}} a_{i}^{\dagger} a_{j}\right)
\end{aligned}
$$

where the couplings are

$$
t^{\mathrm{I}, \mathrm{A},(\mathrm{M}, \mathrm{A})}=V_{p p \pi}+f_{\mathrm{geo}} b^{2}\left(V_{p p \sigma}-V_{p p \pi}\right)+\mathcal{O}\left(\xi_{s p}^{2}\right)
$$

on the inner and outer helix and

$$
t^{\mathrm{I}, \mathrm{M}}=V_{p p \pi}+\mathcal{O}\left(\xi_{s p}^{2}\right)
$$

between the two helices. The geometry-dependent factors are $f_{\text {geo }}^{\mathrm{I}}=f_{\text {geo }}\left(a_{0}, \phi_{0}\right)$ and $f_{\text {geo }}^{\mathrm{A}}=$ $f_{\text {geo }}\left(a_{1}, \phi_{2}\right)$ with

$$
f_{\text {geo }}(a, \phi)=\phi^{2} /\left(8 \pi^{2} a^{2}(1-\cos \phi)+b^{2} \phi^{2}\right)
$$

and $f_{\text {geo }}^{\mathrm{M}, \mathrm{A}}=\phi_{1}^{2} /\left(4 \pi^{2}\left(4 a_{0}^{2}-4 a_{0} a_{1} \cos \phi_{1}+a_{1}^{2}\right)+b^{2} \phi_{1}^{2}\right)$.

Here and in the following, functions and constants with the subscript geo only depend on the three angles $\phi_{1}, \phi_{2}, \phi_{3}$ and three length scales $a_{0}, a_{1}$ and $b$. Couplings on the inner helix $\mathcal{I}$ only depend on the angle $\phi_{0}$ and the lattice constant $a_{0}$. The couplings on the outer helix $\mathcal{A}$ are the same as on $\mathcal{I}$ but with $\phi_{2}$ instead of $\phi_{0}$ and $a_{0}$ replaced with the outer helix radius 
$a_{1}$ since their geometry is similar. These kinds of couplings also appear on the helices of the DNA-Model in Valera et. al. $\stackrel{2}{ }$.

Written out explicitly for all orders, the couplings are

$$
\begin{aligned}
t^{\mathrm{I}} & =\frac{b^{2} \phi_{0}^{2}\left(V_{p p \sigma}-V_{p p \pi}\right)}{8 \pi^{2} a_{0}^{2}\left(1-\cos \phi_{0}\right)+b^{2} \phi_{0}^{2}}+V_{p p \pi}+\lambda_{z}^{\mathrm{I}} \cot \phi_{0}+\rho_{z}^{\mathrm{I}}\left(1-\cos \phi_{0}\right), \\
t^{\mathrm{A}} & =\frac{b^{2} \phi_{2}^{2}\left(V_{p p \sigma}-V_{p p \pi}\right)}{8 \pi^{2} a_{1}^{2}\left(1-\cos \phi_{2}\right)+b^{2} \phi_{2}^{2}}+V_{p p \pi}+\lambda_{z}^{\mathrm{A}} \cot \phi_{2}+\rho_{z}^{\mathrm{A}}\left(1-\cos \phi_{2}\right), \\
t^{\mathrm{I}, \mathrm{M}} & =V_{p p \pi}+\xi_{p}^{2} \frac{\left(V_{p p \pi}+V_{p p \sigma}\right)}{\left(\varepsilon_{p \pi}-\varepsilon_{p \sigma}\right)^{2}}, \\
t^{\mathrm{M}, \mathrm{A}} & =\frac{b^{2} \phi_{1}^{2}\left(V_{p p \sigma}-V_{p p \pi}\right)}{4 \pi^{2}\left(4 a_{0}^{2}-4 a_{0} a_{1} \cos \phi_{1}+a_{1}^{2}\right)+b^{2} \phi_{1}^{2}}+V_{p p \pi}+\lambda_{z}^{\mathrm{M}, \mathrm{A}} \cot \phi_{1} .
\end{aligned}
$$

Here the $\lambda_{z}$ 's and $\rho_{z}$ 's are the $\sigma_{z}$-couplings from the intrinsic SOC and Rashba interaction given in the following subsections. They stem from contributions of second order in $\xi_{p}$ proportional to the identity operator in spin space. The addtional term with prefactor $\xi_{p}^{2}$ in $t^{\mathrm{I}, \mathrm{M}}$ is of the same origin.

\subsection{Intrinsic SOC}

The intrinsic spin-orbit coupling term is

$$
\begin{aligned}
& H_{\mathrm{SOC}}=i \lambda_{y}^{\mathrm{I}} \sum_{(i, j)}^{\mathcal{N}_{\mathcal{I}}} a_{i}^{\dagger} \nu_{i j} \sigma_{y} a_{j}-i \lambda_{z}^{\mathrm{I}} \sum_{(i, j)}^{\mathcal{N}_{\mathcal{I}}} a_{i}^{\dagger} \nu_{i j} \sigma_{z} a_{j} \\
& +i \lambda_{y}^{\mathrm{A}} \sum_{(i, j)}^{\mathcal{N}_{\mathcal{A}}} a_{i}^{\dagger} \nu_{i j} \sigma_{y} a_{j}-i \lambda_{z}^{\mathrm{A}} \sum_{(i, j)}^{\mathcal{N}_{\mathcal{A}}} a_{i}^{\dagger} \nu_{i j} \sigma_{z} a_{j} \\
& +i \lambda_{x}^{\mathrm{M}, \mathrm{A}} \sum_{(i, j)}^{\mathcal{N}_{(\mathcal{M}, \mathcal{A})}} a_{i}^{\dagger} \nu_{i j} \sigma_{x} a_{j}-i \lambda_{x}^{\mathrm{M}, \mathrm{A}} \sum_{(i, j)}^{\mathcal{N}_{(\mathcal{A}, \mathcal{M})}} a_{i}^{\dagger} \nu_{i j} \sigma_{x} a_{j} \\
& +i \lambda_{y}^{\mathrm{M}, \mathrm{A}} \sum_{(i, j)}^{\mathcal{N}_{(\mathcal{M}, \mathcal{A})}^{\cup \mathcal{N}_{(\mathcal{A}, \mathcal{M})}}} a_{i}^{\dagger} \nu_{i j} \sigma_{y} a_{j}-i \lambda_{z}^{\mathrm{M}, \mathrm{A}} \sum_{(i, j)}^{\mathcal{N}_{(\mathcal{M}, \mathcal{A})}^{\cup \mathcal{N}_{(\mathcal{A}, \mathcal{M})}}} a_{i}^{\dagger} \nu_{i j} \sigma_{z} a_{j}
\end{aligned}
$$


with $\nu_{i j}:=\operatorname{sgn}(j-i)$ and

$$
\begin{gathered}
\lambda_{y}^{\mathrm{I}, \mathrm{A},(\mathrm{M}, \mathrm{A})}=\xi_{p} b g_{\mathrm{geo}, y} \frac{V_{p p \pi}-V_{p p \sigma}}{\varepsilon_{p \pi}-\varepsilon_{p \sigma}}+\mathcal{O}\left(\xi_{p}^{2}\right), \\
\lambda_{x}^{\mathrm{M}, \mathrm{A}}=\xi_{p} b g_{\mathrm{geo}, x}^{\mathrm{M}, \mathrm{A}} \frac{V_{p p \pi}-V_{p p \sigma}}{\varepsilon_{p \pi}-\varepsilon_{p \sigma}}+\mathcal{O}\left(\xi_{p}^{2}\right),
\end{gathered}
$$

where $\lambda_{z}^{\mathrm{I}, \mathrm{A},(\mathrm{M}, \mathrm{A})}=\mathcal{O}\left(\xi_{p}^{2}\right)$ and the geometry-dependent factors are $g_{\text {geo }, y}^{\mathrm{I}}=g_{\text {geo }}\left(a_{0}, \phi_{0}\right)$ amd $g_{\text {geo }, y}^{\mathrm{A}}=g_{\text {geo }}\left(a_{1}, \phi_{2}\right)$ with

$$
g_{\text {geo }}(a, \phi)=4 \pi a f_{\text {geo }}(a, \phi)(1-\cos \phi) / \phi
$$

and

$$
\begin{gathered}
g_{\mathrm{geo}, y}^{\mathrm{M}, \mathrm{A}}=2 \pi\left(2 a_{0}+a_{1}\right) f_{\mathrm{geo}}^{\mathrm{M}, \mathrm{A}}\left(1-\cos \phi_{1}\right) / \phi_{1} \\
g_{\mathrm{geo}, x}^{\mathrm{M}, \mathrm{A}}=2 \pi\left(2 a_{0}-a_{1}\right) f_{\mathrm{geo}}^{\mathrm{M}, \mathrm{A}} \sin \phi_{1} / \phi_{1} .
\end{gathered}
$$

Written out fully, they are

$$
\begin{aligned}
\lambda_{y}^{\mathrm{I}} & =\left(\xi_{p}-\frac{\xi_{p}^{2}}{\varepsilon_{p \pi}-\varepsilon_{p \sigma}}\right) \frac{4 \pi a_{0} b \phi_{0}\left(\cos \phi_{0}-1\right)\left(V_{p p \pi}-V_{p p \sigma}\right)}{\left(\varepsilon_{p \pi}-\varepsilon_{p \sigma}\right)\left(8 \pi^{2} a_{0}^{2}\left(1-\cos \phi_{0}\right)+b^{2} \phi_{0}^{2}\right)}, \\
\lambda_{z}^{\mathrm{I}} & =\xi_{p}^{2} 2 \sin \phi_{0} \frac{4 \pi^{2} a_{0}^{2}\left(1-\cos \phi_{0}\right)\left(V_{p p \pi}+V_{p p \sigma}\right)+b^{2} \phi_{0}^{2} V_{p p \pi}}{\left(\varepsilon_{p \pi}-\varepsilon_{p \sigma}\right)^{2}\left(8 \pi^{2} a_{0}^{2}\left(1-\cos \phi_{0}\right)+b^{2} \phi_{0}^{2}\right)} \\
\lambda_{y}^{\mathrm{A}} & =\left(\xi_{p}-\frac{\xi_{p}^{2}}{\varepsilon_{p \pi}-\varepsilon_{p \sigma}}\right) \frac{4 \pi a_{1} b \phi_{2}\left(\cos \phi_{2}-1\right)\left(V_{p p \pi}-V_{p p \sigma}\right)}{\left(\varepsilon_{p \pi}-\varepsilon_{p \sigma}\right)\left(8 \pi^{2} a_{1}^{2}\left(1-\cos \phi_{2}\right)+b^{2} \phi_{2}^{2}\right)} \\
\lambda_{z}^{\mathrm{A}} & =\xi_{p}^{2} 2 \sin \phi_{2} \frac{4 \pi^{2} a_{1}^{2}\left(1-\cos \phi_{2}\right)\left(V_{p p \pi}+V_{p p \sigma}\right)+b^{2} \phi_{2}^{2} V_{p p \pi}}{\left(\varepsilon_{p \pi}-\varepsilon_{p \sigma}\right)^{2}\left(8 \pi^{2} a_{1}^{2}\left(1-\cos \phi_{2}\right)+b^{2} \phi_{2}^{2}\right)}
\end{aligned}
$$

and

$$
\begin{aligned}
\lambda_{y}^{\mathrm{M}, \mathrm{A}} & =\left(\xi_{p}-\frac{\xi_{p}^{2}}{\varepsilon_{p \pi}-\varepsilon_{p \sigma}}\right) \frac{2 \pi b \phi_{1}\left(V_{p p \pi}-V_{p p \sigma}\right)\left(2 a_{0}+a_{1}\right)\left(\cos \phi_{1}-1\right)}{\left(\varepsilon_{p \pi}-\varepsilon_{p \sigma}\right)\left(4 \pi^{2}\left(4 a_{0}^{2}-4 a_{0} a_{1} \cos \phi_{1}+a_{1}^{2}\right)+b^{2} \phi_{1}^{2}\right)}, \\
\lambda_{x}^{\mathrm{M}, \mathrm{A}} & =\left(\xi_{p}-\frac{\xi_{p}^{2}}{\varepsilon_{p \pi}-\varepsilon_{p \sigma}}\right) \frac{2 \pi b \phi_{1}\left(V_{p p \pi}-V_{p p \sigma}\right)\left(2 a_{0}-a_{1}\right) \sin \phi_{1}}{\left(\varepsilon_{p \pi}-\varepsilon_{p \sigma}\right)\left(4 \pi^{2}\left(4 a_{0}^{2}-4 a_{0} a_{1} \cos \phi_{1}+a_{1}^{2}\right)+b^{2} \phi_{1}^{2}\right)} \\
\lambda_{z}^{\mathrm{M}, \mathrm{A}} & =\xi_{p}^{2} 2 \sin \phi_{1} \frac{2 \pi^{2}\left(4 a_{0}^{2}-4 a_{0} a_{1} \cos \phi_{1}+a_{1}^{2}\right)\left(V_{p p \pi}+V_{p p \sigma}\right)+b^{2} \phi_{1}^{2} V_{p p \pi}}{\left(\varepsilon_{p \pi}-\varepsilon_{p \sigma}\right)^{2}\left(4 \pi^{2}\left(4 a_{0}^{2}-4 a_{0} a_{1} \cos \phi_{1}+a_{1}^{2}\right)+b^{2} \phi_{1}^{2}\right)}
\end{aligned}
$$




\subsection{Rashba, first order terms}

Up to first order in $\xi_{s p}$, the Rashba term, containing all effective couplings arising from a combination of SOC and external field is

$$
\begin{aligned}
H_{\text {Rashba }} & =i \rho_{x}^{\mathrm{I}} \sum_{(i, j)}^{\mathcal{N}_{\mathcal{I}}} a_{i}^{\dagger} \nu_{i j} \sigma_{x} a_{j}+i \rho_{y}^{\mathrm{I}} \sum_{(i, j)}^{\mathcal{N}_{\mathcal{I}}} a_{i}^{\dagger} \nu_{i j} \sigma_{y} a_{j}+i \rho_{z}^{\mathrm{I}} \sum_{(i, j)}^{\mathcal{N}_{\mathcal{I}}} a_{i}^{\dagger} \nu_{i j} \sigma_{z} a_{j} \\
& +i \rho_{x}^{\mathrm{A}} \sum_{(i, j)}^{\mathcal{N}_{\mathcal{A}}} a_{i}^{\dagger} \nu_{i j} \sigma_{x} a_{j}+i \rho_{y}^{\mathrm{A}} \sum_{(i, j)}^{\mathcal{N}_{\mathcal{A}}} a_{i}^{\dagger} \nu_{i j} \sigma_{y} a_{j}+i \rho_{z}^{\mathrm{A}} \sum_{(i, j)}^{\mathcal{N}_{\mathcal{A}}} a_{i}^{\dagger} \nu_{i j} \sigma_{z} a_{j} \\
& +i \rho_{y}^{\mathrm{I}, \mathrm{M}} \sum_{(i, j)}^{\mathcal{N}_{(\mathcal{I}, \mathcal{M})}} a_{i}^{\dagger} \nu_{i j} \sigma_{y} a_{j}+i \rho_{z}^{\mathrm{I}, \mathrm{M}} \sum_{(i, j)}^{\mathcal{N}_{(\mathcal{I}, \mathcal{M})}} a_{i}^{\dagger} \nu_{i j} \sigma_{z} a_{j} \\
& +i \rho_{x}^{\mathrm{M}, \mathrm{A}} \sum_{\mathcal{N}_{(\mathcal{M}, \mathcal{A})}^{\cup \mathcal{N}}\left(\mathcal{N}_{(\mathcal{A}, \mathcal{M})}\right.} a_{i}^{\dagger} \nu_{i j} \sigma_{x} a_{j}+i \rho_{y}^{\mathrm{M}, \mathrm{A}} \sum_{(i, j)}^{\mathcal{N}_{(\mathcal{M}, \mathcal{A})}} a_{i}^{\dagger} \nu_{i j} \sigma_{y} a_{j} \\
& -i \rho_{y}^{\mathrm{A}, \mathrm{M}} \sum_{(i, j)}^{\mathcal{N}_{(\mathcal{A}, \mathcal{M})}} a_{i}^{\dagger} \nu_{i j} \sigma_{y} a_{j}+i \rho_{z}^{\mathrm{M}, \mathrm{A}} \sum_{(i, j)}^{\mathcal{N}_{(\mathcal{M}, \mathcal{A})}} a_{i}^{\dagger} \nu_{i j} \sigma_{z} a_{j}+i \rho_{z}^{\mathrm{A}, \mathrm{M}} \sum_{(i, j)}^{\mathcal{N}_{(\mathcal{A}, \mathcal{M})}} a_{i}^{\dagger} \nu_{i j} \sigma_{z} a_{j}
\end{aligned}
$$

with $\nu_{i j}:=\operatorname{sgn}(j-i)$,

$$
\begin{aligned}
& \rho_{x}^{\mathrm{I}}=-\left(\xi_{p}-\frac{\xi_{p}^{2}}{\varepsilon_{p \pi}-\varepsilon_{p \sigma}}\right) \frac{4 \pi V_{s p \sigma}\left(2 \pi a_{0} E_{z} \sin \phi_{0}-b \phi_{0} E_{\mathrm{t}}\right)}{\left(\varepsilon_{p \pi}-\varepsilon_{p \sigma}\right)\left(\varepsilon_{p \pi}-\varepsilon_{s}\right) \sqrt{8 \pi^{2} a_{0}^{2}\left(1-\cos \phi_{0}\right)+b^{2} \phi_{0}^{2}}}, \\
& \rho_{y}^{\mathrm{I}}=-\left(\xi_{p}-\frac{\xi_{p}^{2}}{\varepsilon_{p \pi}-\varepsilon_{p \sigma}}\right) \frac{4 \pi V_{s p \sigma} b \phi_{0} E_{\mathrm{n}}}{\left(\varepsilon_{p \pi}-\varepsilon_{p \sigma}\right)\left(\varepsilon_{p \pi}-\varepsilon_{s}\right) \sqrt{8 \pi^{2} a_{0}^{2}\left(1-\cos \phi_{0}\right)+b^{2} \phi_{0}^{2}}}, \\
& \rho_{z}^{\mathrm{I}}=\xi_{p}^{2} \frac{8 \pi^{2} a_{0} V_{s p \sigma} E_{\mathrm{n}} \sin \phi_{0}}{\left(\varepsilon_{p \pi}-\varepsilon_{p \sigma}\right)^{2}\left(\varepsilon_{p \pi}-\varepsilon_{s}\right) \sqrt{8 \pi^{2} a_{0}^{2}\left(1-\cos \phi_{0}\right)+b^{2} \phi_{0}^{2}}}, \\
& \rho_{x}^{\mathrm{A}}=-\left(\xi_{p}-\frac{\xi_{p}^{2}}{\varepsilon_{p \pi}-\varepsilon_{p \sigma}}\right) \frac{4 \pi V_{s p \sigma}\left(2 \pi a_{1} E_{z} \sin \phi_{2}-b \phi_{2} E_{\mathrm{t}}\right)}{\left(\varepsilon_{p \pi}-\varepsilon_{p \sigma}\right)\left(\varepsilon_{p \pi}-\varepsilon_{s}\right) \sqrt{8 \pi^{2} a_{1}^{2}\left(1-\cos \phi_{2}\right)+b^{2} \phi_{2}^{2}}}, \\
& \rho_{y}^{\mathrm{A}}=-\left(\xi_{p}-\frac{\xi_{p}^{2}}{\varepsilon_{p \pi}-\varepsilon_{p \sigma}}\right) \frac{4 \pi V_{s p \sigma} b \phi_{2} E_{\mathrm{n}}}{\left(\varepsilon_{p \pi}-\varepsilon_{p \sigma}\right)\left(\varepsilon_{p \pi}-\varepsilon_{s}\right) \sqrt{8 \pi^{2} a_{1}^{2}\left(1-\cos \phi_{2}\right)+b^{2} \phi_{2}^{2}}}, \\
& \rho_{z}^{\mathrm{A}}=\xi_{p}^{2} \frac{8 \pi^{2} a_{1} V_{s p \sigma} E_{\mathrm{n}} \sin \phi_{2}}{\left(\varepsilon_{p \pi}-\varepsilon_{p \sigma}\right)^{2}\left(\varepsilon_{p \pi}-\varepsilon_{s}\right) \sqrt{8 \pi^{2} a_{1}^{2}\left(1-\cos \phi_{2}\right)+b^{2} \phi_{2}^{2}}}
\end{aligned}
$$


and

$$
\begin{aligned}
\rho_{y}^{\mathrm{I}, \mathrm{M}} & =-\left(\xi_{p}-\frac{\xi_{p}^{2}}{\varepsilon_{p \pi}-\varepsilon_{p \sigma}}\right) \frac{2 E_{z} V_{s p \sigma}}{\left(\varepsilon_{p \pi}-\varepsilon_{p \sigma}\right)\left(\varepsilon_{p \pi}-\varepsilon_{s}\right)}, \\
\rho_{z}^{\mathrm{I}, \mathrm{M}} & =\xi_{p}^{2} \frac{2 E_{\mathrm{t}} V_{s p \sigma}}{\left(\varepsilon_{p \pi}-\varepsilon_{p \sigma}\right)^{2}\left(\varepsilon_{p \pi}-\varepsilon_{s}\right)}, \\
\rho_{x}^{\mathrm{M}, \mathrm{A}} & =-\left(\xi_{p}-\frac{\xi_{p}^{2}}{\varepsilon_{p \pi}-\varepsilon_{p \sigma}}\right) \frac{4 \pi V_{s p \sigma}}{\left(\varepsilon_{p \pi}-\varepsilon_{p \sigma}\right)\left(\varepsilon_{p \pi}-\varepsilon_{s}\right)} \\
& \times \frac{\pi E_{z}\left(2 a_{0}+a_{1}\right) \sin \phi_{1}-E_{\mathrm{t}} b \phi_{1}}{\sqrt{4 \pi^{2}\left(4 a_{0}^{2}-4 a_{0} a_{1} \cos \phi_{1}+a_{1}^{2}\right)+b^{2} \phi_{1}^{2}}}, \\
\rho_{y}^{\mathrm{M}, \mathrm{A}} & =-\left(\xi_{p}-\frac{\xi_{p}^{2}}{\varepsilon_{p \pi}-\varepsilon_{p \sigma}}\right) \frac{4 \pi V_{s p \sigma}}{\left(\varepsilon_{p \pi}-\varepsilon_{p \sigma}\right)\left(\varepsilon_{p \pi}-\varepsilon_{s}\right)} \\
& \times \frac{\pi E_{z}\left(2 a_{0}-a_{1}\right)\left(1+\cos \phi_{1}\right)+E_{\mathrm{n}} b \phi_{1}}{\sqrt{4 \pi^{2}\left(4 a_{0}^{2}-4 a_{0} a_{1} \cos \phi_{1}+a_{1}^{2}\right)+b^{2} \phi_{1}^{2}}}, \\
\rho_{y}^{\mathrm{A}, \mathrm{M}} & =-\left(\xi_{p}-\frac{\xi_{p}^{2}}{\varepsilon_{p \pi}-\varepsilon_{p \sigma}}\right) \frac{4 \pi V_{s p \sigma}}{\left(\varepsilon_{p \pi}-\varepsilon_{p \sigma}\right)\left(\varepsilon_{p \pi}-\varepsilon_{s}\right)} \\
& \times \frac{\pi E_{z}\left(2 a_{0}-a_{1}\right)\left(1+\cos \phi_{1}\right)-E_{\mathrm{n}} b \phi_{1}}{\sqrt{4 \pi^{2}\left(4 a_{0}^{2}-4 a_{0} a_{1} \cos \phi_{1}+a_{1}^{2}\right)+b^{2} \phi_{1}^{2}}}, \\
\rho_{z}^{\mathrm{M}, \mathrm{A}} & =\xi_{p}^{2} \frac{2 \pi V_{s p \sigma}\left(E_{\mathrm{t}}\left(2 a_{0}-a_{1}\right)\left(1+\cos \phi_{1}\right)+E_{\mathrm{n}}\left(2 a_{0}+a_{1}\right) \sin \phi_{1}\right)}{\left(\varepsilon_{p \pi}-\varepsilon_{p \sigma}\right)^{2}\left(\varepsilon_{p \pi}-\varepsilon_{s}\right) \sqrt{4 \pi^{2}\left(4 a_{0}^{2}-4 a_{0} a_{1} \cos \phi_{1}+a_{1}^{2}\right)+b^{2} \phi_{1}^{2}}}, \\
= & \xi_{p}^{2} \frac{2 \pi V_{s p \sigma}\left(-E_{\mathrm{t}}\left(2 a_{0}-a_{1}\right)\left(1+\cos \phi_{1}\right)+E_{\mathrm{n}}\left(2 a_{0}+a_{1}\right) \sin \phi_{1}\right)}{\left(\varepsilon_{p \pi}-\varepsilon_{p \sigma}\right)^{2}\left(\varepsilon_{p \pi}-\varepsilon_{s}\right) \sqrt{4 \pi^{2}\left(4 a_{0}^{2}-4 a_{0} a_{1} \cos \phi_{1}+a_{1}^{2}\right)+b^{2} \phi_{1}^{2}}}
\end{aligned}
$$

\subsection{Rashba, second order terms}

Since the expressions for the second order terms in $\xi_{s p}$ are somewhat lengthy they shall be treated independently from the rest. Both terms proportional to the Pauli matrices and diagonal in spin-space (which are corrections to the couplings in the kinetic term) arise. They shall be listed in the following.

The correction to the kinetic Hamiltonian is

$$
H_{\mathrm{kin}, \mathrm{R} 2}=t_{\mathrm{R} 2}^{\mathrm{I}} \sum_{(i, j)}^{\mathcal{N}_{\mathcal{I}}} a_{i}^{\dagger} a_{j}+t_{\mathrm{R} 2}^{\mathrm{A}} \sum_{(i, j)}^{\mathcal{N}_{\mathcal{A}}} a_{i}^{\dagger} a_{j}+t_{\mathrm{R} 2}^{\mathrm{I}, \mathrm{M}} \sum_{(i, j)}^{\mathcal{N}_{(\mathcal{I}, \mathcal{M})}} a_{i}^{\dagger} a_{j}+t_{\mathrm{R} 2}^{\mathrm{M}, \mathrm{A}} \sum_{(i, j)}^{\mathcal{N}_{(\mathcal{M}, \mathcal{A})}} a_{i}^{\dagger} a_{j}+t_{\mathrm{R} 2}^{\mathrm{A}, \mathrm{M}} \sum_{(i, j)}^{\mathcal{N}_{(\mathcal{A}, \mathcal{M})}} a_{i}^{\dagger} a_{j}
$$


with

$$
\begin{aligned}
t_{\mathrm{R} 2}^{\mathrm{I}} & =-\frac{4 \pi a_{0} b E_{\mathrm{t}} E_{z} \phi_{0}\left(V_{p p \pi}-V_{p p \sigma}\right) \sin \phi_{0}}{\left(8 \pi^{2} a_{0}^{2}\left(1-\cos \phi_{0}\right)+b^{2} \phi_{0}^{2}\right)\left(\varepsilon_{p \pi}-\varepsilon_{p \sigma}\right)\left(\varepsilon_{p \pi}-\varepsilon_{s}\right)}-\frac{E_{z}^{2} V_{s s}}{\left(\varepsilon_{p \pi}-\varepsilon_{s}\right)^{2}} \\
& +\frac{\xi_{p}^{2}}{\left(8 \pi^{2} a_{0}^{2}\left(1-\cos \phi_{0}\right)+b^{2} \phi_{0}^{2}\right)\left(\varepsilon_{p \pi}-\varepsilon_{s}\right)\left(\varepsilon_{p \pi}-\varepsilon_{p \sigma}\right)^{3}}\left(4 \pi ^ { 2 } a _ { 0 } ^ { 2 } \left(\left(4 \cos \phi_{0}-\cos \left(2 \phi_{0}\right)-3\right) \times\right.\right. \\
& \left.\times\left(E_{\mathrm{t}}^{2} V_{p p \pi}+E_{\mathrm{n}}^{2} V_{p p \sigma}\right)+2\left(\sin \phi_{0}\right)^{2}\left(E_{\mathrm{t}}^{2} V_{p p \sigma}+E_{\mathrm{n}}^{2} V_{p p \pi}\right)\right)-4 \pi a_{0} b E_{\mathrm{t}} E_{z} \sin \phi_{0} \phi_{0}\left(V_{p p \pi}-V_{p p \sigma}\right) \\
& \left.+\left(E_{\mathrm{t}}^{2}+E_{\mathrm{n}}^{2}\right) 2 b^{2} \cos \phi_{0} \phi_{0}^{2} V_{p p \pi}\right)-\frac{\xi_{p}^{2}\left(E_{\mathrm{t}}^{2}+E_{\mathrm{n}}^{2}\right) V_{s s}}{\left(\varepsilon_{p \pi}-\varepsilon_{s}\right)^{2}\left(\varepsilon_{p \pi}-\varepsilon_{p \sigma}\right)^{2}}, \\
t_{\mathrm{R} 2}^{\mathrm{A}} & =-\frac{4 \pi a_{1} b E_{\mathrm{t}} E_{z} \phi_{2}\left(V_{p p \pi}-V_{p p \sigma}\right) \sin \phi_{2}}{\left(8 \pi^{2} a_{1}^{2}\left(1-\cos \phi_{2}\right)+b^{2} \phi_{2}^{2}\right)\left(\varepsilon_{p \pi}-\varepsilon_{p \sigma}\right)\left(\varepsilon_{p \pi}-\varepsilon_{s}\right)}-\frac{E_{z}^{2} V_{s s}}{\left(\varepsilon_{p \pi}-\varepsilon_{s}\right)^{2}} \\
& +\frac{\xi_{p}^{2}}{\left(8 \pi^{2} a_{1}^{2}\left(1-\cos \phi_{2}\right)+b^{2} \phi_{2}^{2}\right)\left(\varepsilon_{p \pi}-\varepsilon_{s}\right)\left(\varepsilon_{p \pi}-\varepsilon_{p \sigma}\right)^{3}}\left(4 \pi ^ { 2 } a _ { 1 } ^ { 2 } \left(\left(4 \cos \phi_{2}-\cos \left(2 \phi_{2}\right)-3\right) \times\right.\right. \\
& \left.\times\left(E_{\mathrm{t}}^{2} V_{p p \pi}+E_{\mathrm{n}}^{2} V_{p p \sigma}\right)+2\left(\sin \phi_{2}\right)^{2}\left(E_{\mathrm{t}}^{2} V_{p p \sigma}+E_{\mathrm{n}}^{2} V_{p p \pi}\right)\right)-4 \pi a_{1} b E_{\mathrm{t}} E_{z} \sin \phi_{2} \phi_{2}\left(V_{p p \pi}-V_{p p \sigma}\right) \\
& \left.+\left(E_{\mathrm{t}}^{2}+E_{\mathrm{n}}^{2}\right) 2 b^{2} \cos \phi_{2} \phi_{2}^{2} V_{p p \pi}\right)-\frac{\xi_{p}^{2}\left(E_{\mathrm{t}}^{2}+E_{\mathrm{n}}^{2}\right) V_{s s}}{\left(\varepsilon_{p \pi}-\varepsilon_{s}\right)^{2}\left(\varepsilon_{p \pi}-\varepsilon_{p \sigma}\right)^{2}}, \\
& t_{\mathrm{R} 2}^{\mathrm{I}, \mathrm{M}}=-\frac{E_{z}^{2} V_{s s}}{\left(\varepsilon_{p \pi}-\varepsilon_{s}\right)^{2}}+\xi_{p}^{2} \frac{\left(2\left(\varepsilon_{p \pi}-\varepsilon_{s}\right)\left(E_{\mathrm{t}}^{2} V_{p p \pi}+E_{\mathrm{n}}^{2} V_{p p \sigma}\right)+V_{s s}\left(E_{\mathrm{t}}^{2}+E_{\mathrm{n}}^{2}\right)\left(\varepsilon_{p \sigma}-\varepsilon_{p \pi}\right)\right)}{\left(\varepsilon_{p \pi}-\varepsilon_{p \sigma}\right)^{3}\left(\varepsilon_{p \pi}-\varepsilon_{s}\right)^{2}}
\end{aligned}
$$

and

$$
\begin{aligned}
t_{\mathrm{R} 2}^{\mathrm{M}, \mathrm{A}} & =\frac{2 \pi b E_{z} \phi_{1}\left(V_{p p \pi}-V_{p p \sigma}\right)\left(-\left(2 a_{0}+a_{1}\right) E_{\mathrm{t}} \sin \phi_{1}+\left(2 a_{0}-a_{1}\right) E_{\mathrm{n}}\left(1+\cos \phi_{1}\right)\right)}{\left(4 \pi^{2}\left(4 a_{0}^{2}-4 a_{0} a_{1} \cos \phi_{1}+a_{1}^{2}\right)+b^{2} \phi_{1}^{2}\right)\left(\varepsilon_{p \pi}-\varepsilon_{s}\right)\left(\varepsilon_{p \pi}-\varepsilon_{p \sigma}\right)} \\
& -\frac{4 \pi^{2} E_{z}^{2} V_{s s}}{\left(\varepsilon_{p \pi}-\varepsilon_{s}\right)^{2}} \\
& +\frac{\xi_{p}^{2}}{\left(4 \pi^{2}\left(4 a_{0}^{2}-4 a_{0} a_{1} \cos \phi_{1}+a_{1}^{2}\right)+b^{2} \phi_{1}^{2}\right)\left(\varepsilon_{p \pi}-\varepsilon_{s}\right)\left(\varepsilon_{p \pi}-\varepsilon_{p \sigma}\right)^{3}}\left(8 \pi^{2}\left(4 a_{0}^{2}+a_{1}^{2}\right) \cos \phi_{1} \times\right. \\
& \times\left(E_{\mathrm{t}}^{2} V_{p p \pi}+E_{\mathrm{n}}^{2} V_{p p \sigma}\right)-8 \pi^{2} a_{0} a_{1}\left(E_{\mathrm{t}}^{2}\left(3 V_{p p \pi}-V_{p p \sigma}\right)+E_{\mathrm{n}}^{2}\left(V_{p p \pi}-3 V_{p p \sigma}\right)\right) \\
& -8 \pi^{2}\left(E_{\mathrm{t}}^{2}+E_{\mathrm{n}}^{2}\right)\left(V_{p p \pi}+V_{p p \sigma}\right) a_{0} a_{1} \cos \left(2 \phi_{1}\right) \\
& +\left(V_{p p \pi}-V_{p p \sigma}\right)\left(\left(2 a_{0}-a_{1}\right)\left(8 \pi^{2}\left(2 a_{0}+a_{1}\right) E_{\mathrm{t}} E_{\mathrm{n}} \sin \phi_{1}+2 \pi b E_{\mathrm{n}} E_{z} \phi_{1}\left(\cos \phi_{1}+1\right)\right)\right. \\
& \left.-2 \pi\left(2 a_{0}+a_{1}\right) b E_{\mathrm{t}} E_{z} \phi_{1} \sin \phi_{1}\right) \\
& \left.+\frac{2 b^{2}\left(E_{\mathrm{t}}^{2}+E_{\mathrm{n}}^{2}\right) \cos \phi_{1} \phi_{1}^{2} V_{p p \pi}\left(\varepsilon_{p \pi}-\varepsilon_{s}\right)}{\left(\varepsilon_{p \pi}-\varepsilon_{s}\right)}\right)-\xi_{p}^{2} \frac{4 \pi^{2}\left(E_{\mathrm{t}}^{2}+E_{\mathrm{n}}^{2}\right) V_{s s}}{\left(\varepsilon_{p \pi}-\varepsilon_{s}\right)^{2}\left(\varepsilon_{p \pi}-\varepsilon_{p \sigma}\right)^{2}}
\end{aligned}
$$


as well as

$$
\begin{aligned}
t_{\mathrm{R} 2}^{\mathrm{A}, \mathrm{M}} & =t_{\mathrm{R} 2}^{\mathrm{M}, \mathrm{A}}-2 \frac{2 \pi\left(V_{p p \pi}-V_{p p \sigma}\right)\left(2 a_{0}-a_{1}\right) b E_{\mathrm{n}} E_{z} \phi_{1}\left(\cos \phi_{1}+1\right)}{\left(4 \pi^{2}\left(4 a_{0}^{2}-4 a_{0} a_{1} \cos \phi_{1}+a_{1}^{2}\right)+b^{2} \phi_{1}^{2}\right)\left(\varepsilon_{p \pi}-\varepsilon_{s}\right)\left(\varepsilon_{p \pi}-\varepsilon_{p \sigma}\right)^{3}} \\
& -2 \xi_{p}^{2} \frac{\left(V_{p p \pi}-V_{p p \sigma}\right) E_{\mathrm{n}}\left(\left(2 a_{0}-a_{1}\right)\left(8 \pi^{2}\left(2 a_{0}+a_{1}\right) E_{\mathrm{t}} \sin \phi_{1}+2 \pi b E_{z} \phi_{1}\left(\cos \phi_{1}+1\right)\right)\right.}{(.}
\end{aligned}
$$

The terms proprtional to one of the Pauli matrices are again collected in

$$
\begin{aligned}
H_{\text {Rashba } 2} & =i \rho_{2, x}^{\mathrm{I}} \sum_{(i, j)}^{\mathcal{N}_{\mathcal{I}}} a_{i}^{\dagger} \nu_{i j} \sigma_{x} a_{j}+i \rho_{2, y}^{\mathrm{I}} \sum_{(i, j)}^{\mathcal{N}_{\mathcal{I}}} a_{i}^{\dagger} \nu_{i j} \sigma_{y} a_{j}+i \rho_{2, z}^{\mathrm{I}} \sum_{(i, j)}^{\mathcal{N}_{\mathcal{I}}} a_{i}^{\dagger} \nu_{i j} \sigma_{z} a_{j} \\
& +i \rho_{2, x}^{\mathrm{A}} \sum_{(i, j)}^{\mathcal{N}_{\mathcal{A}}} a_{i}^{\dagger} \nu_{i j} \sigma_{x} a_{j}+i \rho_{2, y}^{\mathrm{A}} \sum_{(i, j)}^{\mathcal{N}_{\mathcal{A}}} a_{i}^{\dagger} \nu_{i j} \sigma_{y} a_{j}+i \rho_{2, z}^{\mathrm{A}} \sum_{(i, j)}^{\mathcal{N}_{\mathcal{A}}} a_{i}^{\dagger} \nu_{i j} \sigma_{z} a_{j} \\
& +i \rho_{2, x}^{\mathrm{M}, \mathrm{A}} \sum_{(i, j)}^{\mathcal{N}_{(\mathcal{M}, \mathcal{A})}} a_{i}^{\dagger} \nu_{i j} \sigma_{x} a_{j}+i \rho_{2, x}^{\mathrm{A}, \mathrm{M}} \sum_{(i, j)}^{\mathcal{N}_{(\mathcal{A}, \mathcal{M})}} a_{i}^{\dagger} \nu_{i j} \sigma_{x} a_{j} \\
& +i \rho_{2, y}^{\mathrm{M}, \mathrm{A}} \sum_{(i, j)}^{\mathcal{N}_{(\mathcal{M}, \mathcal{A})}} a_{i}^{\dagger} \nu_{i j} \sigma_{x} a_{j}+i \rho_{2, y}^{\mathrm{A}, \mathrm{M}} \sum_{(i, j)}^{\mathcal{N}_{(\mathcal{A}, \mathcal{M})}} a_{i}^{\dagger} \nu_{i j} \sigma_{x} a_{j} \\
& +i \rho_{2, z}^{\mathrm{M}, \mathrm{A}} \sum_{(i, j)}^{\mathcal{N}_{(\mathcal{M}, \mathcal{A})}} a_{i}^{\dagger} \nu_{i j} \sigma_{x} a_{j}+i \rho_{2, z}^{\mathrm{A}, \mathrm{M}} \sum_{(i, j)}^{\mathcal{N}_{(\mathcal{A}, \mathcal{M})}} a_{i}^{\dagger} \nu_{i j} \sigma_{x} a_{j}
\end{aligned}
$$

with

$$
\begin{aligned}
\rho_{2, x}^{\mathrm{I}} & =\xi_{p}\left(\frac{2 E_{\mathrm{n}} E_{z} \sin \phi_{0}\left(4 \pi^{2} a_{0}^{2}\left(V_{p p \pi}+V_{p p \sigma}\right)\left(1-\cos \phi_{0}\right)+b^{2} \phi_{0}^{2} V_{p p \pi}\right)}{\left(8 \pi^{2} a_{0}^{2}\left(1-\cos \phi_{0}\right)+b^{2} \phi_{0}^{2}\right)\left(\varepsilon_{p \pi}-\varepsilon_{s}\right)\left(\varepsilon_{p \pi}-\varepsilon_{p \sigma}\right)^{2}}\right. \\
& \left.+\frac{4 \pi a_{0} b E_{\mathrm{t}} E_{\mathrm{n}} \phi_{0}\left(V_{p p \pi}-V_{p p \sigma}\right)\left(1-\cos \phi_{0}\right)}{\left(8 \pi^{2} a_{0}^{2}\left(1-\cos \phi_{0}\right)+b^{2} \phi_{0}^{2}\right)\left(\varepsilon_{p \pi}-\varepsilon_{s}\right)\left(\varepsilon_{p \pi}-\varepsilon_{p \sigma}\right)^{2}}\right) \\
& -\frac{\xi_{p}^{2}}{\left(\varepsilon_{p \pi}-\varepsilon_{p \sigma}\right)}\left(4 E_{\mathrm{n}} E_{z} \sin \phi_{0} \frac{\pi^{2} a_{0}^{2}\left(V_{p p \pi}+V_{p p \sigma}\right)\left(1-\cos \phi_{0}\right)+b^{2} \phi_{0}^{2} V_{p p \pi}}{\left(8 \pi^{2} a_{0}^{2}\left(1-\cos \phi_{0}\right)+b^{2} \phi_{0}^{2}\right)\left(\varepsilon_{p \pi}-\varepsilon_{s}\right)\left(\varepsilon_{p \pi}-\varepsilon_{p \sigma}\right)^{2}}\right),
\end{aligned}
$$




$$
\begin{aligned}
& \rho_{2, y}^{\mathrm{I}}=\xi_{p}\left(\frac{2 E_{\mathrm{t}} E_{z} \sin \phi_{0}\left(4 \pi^{2} a_{0}^{2}\left(V_{p p \pi}+V_{p p \sigma}\right)\left(1-\cos \phi_{0}\right)+b^{2} \phi_{0}^{2} V_{p p \pi}\right)}{\left(8 \pi^{2} a_{0}^{2}\left(1-\cos \phi_{0}\right)+b^{2} \phi_{0}^{2}\right)\left(\varepsilon_{p \pi}-\varepsilon_{s}\right)\left(\varepsilon_{p \pi}-\varepsilon_{p \sigma}\right)^{2}}\right. \\
& \left.-\frac{4 \pi a_{0} b E_{\mathrm{n}}^{2} \phi_{0}\left(V_{p p \pi}-V_{p p \sigma}\right)\left(1-\cos \phi_{0}\right)}{\left(8 \pi^{2} a_{0}^{2}\left(1-\cos \phi_{0}\right)+b^{2} \phi_{0}^{2}\right)\left(\varepsilon_{p \pi}-\varepsilon_{s}\right)\left(\varepsilon_{p \pi}-\varepsilon_{p \sigma}\right)^{2}}\right) \\
& +\frac{\xi_{p}^{2}}{\left(\varepsilon_{p \pi}-\varepsilon_{p \sigma}\right)}\left(\frac{-4 E_{\mathrm{t}} E_{z} \sin \phi_{0}\left(4 \pi^{2} a_{0}^{2}\left(V_{p p \pi}+V_{p p \sigma}\right)\left(1-\cos \phi_{0}\right)+b^{2} \phi_{0}^{2} V_{p p \pi}\right)}{\left(8 \pi^{2} a_{0}^{2}\left(1-\cos \phi_{0}\right)+b^{2} \phi_{0}^{2}\right)\left(\varepsilon_{p \pi}-\varepsilon_{s}\right)\left(\varepsilon_{p \pi}-\varepsilon_{p \sigma}\right)^{2}}\right. \\
& \left.+\frac{4 \pi a_{0} b\left(E_{\mathrm{t}}^{2}+E_{\mathrm{n}}^{2}\right) \phi_{0}\left(V_{p p \pi}-V_{p p \sigma}\right)\left(1-\cos \phi_{0}\right)}{\left(8 \pi^{2} a_{0}^{2}\left(1-\cos \phi_{0}\right)+b^{2} \phi_{0}^{2}\right)\left(\varepsilon_{p \pi}-\varepsilon_{s}\right)\left(\varepsilon_{p \pi}-\varepsilon_{p \sigma}\right)^{2}}\right), \\
& \rho_{2, z}^{\mathrm{I}}=\xi_{p}\left(\frac{4 \pi a_{0} b E_{\mathrm{t}} E_{z} \phi_{0}\left(V_{p p \pi}-V_{p p \sigma}\right)\left(1-\cos \phi_{0}\right)}{\left(8 \pi^{2} a_{0}^{2}\left(1-\cos \phi_{0}\right)+b^{2} \phi_{0}^{2}\right)\left(\varepsilon_{p \pi}-\varepsilon_{s}\right)\left(\varepsilon_{p \pi}-\varepsilon_{p \sigma}\right)^{2}}\right) \\
& -\xi_{p}^{2}\left(\frac{8 \pi^{2} a_{0}^{2} \sin \phi_{0}\left(1-\cos \phi_{0}\right)\left(E_{\mathrm{t}}^{2}-E_{\mathrm{n}}^{2}\right)\left(V_{p p \sigma}-V_{p p \pi}\right)+2 b^{2}\left(E_{\mathrm{t}}^{2}+E_{\mathrm{n}}^{2}\right) \sin \phi_{0} \phi_{0}^{2} V_{p p \pi}}{\left(8 \pi^{2} a_{0}^{2}\left(1-\cos \phi_{0}\right)+b^{2} \phi_{0}^{2}\right)\left(\varepsilon_{p \pi}-\varepsilon_{s}\right)\left(\varepsilon_{p \pi}-\varepsilon_{p \sigma}\right)^{3}}\right) \text {, } \\
& \rho_{2, x}^{\mathrm{A}}=\xi_{p}\left(\frac{2 E_{\mathrm{n}} E_{z} \sin \phi_{2}\left(4 \pi^{2} a_{1}^{2}\left(V_{p p \pi}+V_{p p \sigma}\right)\left(1-\cos \phi_{2}\right)+b^{2} \phi_{2}^{2} V_{p p \pi}\right)}{\left(8 \pi^{2} a_{1}^{2}\left(1-\cos \phi_{2}\right)+b^{2} \phi_{2}^{2}\right)\left(\varepsilon_{p \pi}-\varepsilon_{s}\right)\left(\varepsilon_{p \pi}-\varepsilon_{p \sigma}\right)^{2}}\right. \\
& \left.+\frac{4 \pi a_{1} b E_{\mathrm{t}} E_{\mathrm{n}} \phi_{2}\left(V_{p p \pi}-V_{p p \sigma}\right)\left(1-\cos \phi_{2}\right)}{\left(8 \pi^{2} a_{1}^{2}\left(1-\cos \phi_{2}\right)+b^{2} \phi_{2}^{2}\right)\left(\varepsilon_{p \pi}-\varepsilon_{s}\right)\left(\varepsilon_{p \pi}-\varepsilon_{p \sigma}\right)^{2}}\right) \\
& -\frac{\xi_{p}^{2}}{\left(\varepsilon_{p \pi}-\varepsilon_{p \sigma}\right)}\left(4 E_{\mathrm{n}} E_{z} \sin \phi_{2} \frac{4 \pi^{2} a_{1}^{2}\left(V_{p p \pi}+V_{p p \sigma}\right)\left(1-\cos \phi_{2}\right)+b^{2} \phi_{2}^{2} V_{p p \pi}}{\left(8 \pi^{2} a_{1}^{2}\left(1-\cos \phi_{2}\right)+b^{2} \phi_{2}^{2}\right)\left(\varepsilon_{p \pi}-\varepsilon_{s}\right)\left(\varepsilon_{p \pi}-\varepsilon_{p \sigma}\right)^{2}}\right), \\
& \rho_{2, y}^{\mathrm{A}}=\xi_{p}\left(\frac{2 E_{\mathrm{t}} E_{z} \sin \phi_{2}\left(4 \pi^{2} a_{1}^{2}\left(V_{p p \pi}+V_{p p \sigma}\right)\left(1-\cos \phi_{2}\right)+b^{2} \phi_{2}^{2} V_{p p \pi}\right)}{\left(8 \pi^{2} a_{1}^{2}\left(1-\cos \phi_{2}\right)+b^{2} \phi_{2}^{2}\right)\left(\varepsilon_{p \pi}-\varepsilon_{s}\right)\left(\varepsilon_{p \pi}-\varepsilon_{p \sigma}\right)^{2}}\right. \\
& \left.-\frac{4 \pi a_{1} b E_{\mathrm{n}}^{2} \phi_{2}\left(V_{p p \pi}-V_{p p \sigma}\right)\left(1-\cos \phi_{2}\right)}{\left(8 \pi^{2} a_{1}^{2}\left(1-\cos \phi_{2}\right)+b^{2} \phi_{2}^{2}\right)\left(\varepsilon_{p \pi}-\varepsilon_{s}\right)\left(\varepsilon_{p \pi}-\varepsilon_{p \sigma}\right)^{2}}\right) \\
& +\xi_{p}^{2}\left(\frac{-4 E_{\mathrm{t}} E_{z} \sin \phi_{2}\left(4 \pi^{2} a_{1}^{2}\left(V_{p p \pi}+V_{p p \sigma}\right)\left(1-\cos \phi_{2}\right)+b^{2} \phi_{2}^{2} V_{p p \pi}\right)}{\left(8 \pi^{2} a_{1}^{2}\left(1-\cos \phi_{2}\right)+b^{2} \phi_{2}^{2}\right)\left(\varepsilon_{p \pi}-\varepsilon_{s}\right)\left(\varepsilon_{p \pi}-\varepsilon_{p \sigma}\right)^{3}}\right. \\
& \left.+\frac{4 \pi a_{1} b\left(E_{\mathrm{t}}^{2}+E_{\mathrm{n}}^{2}\right) \phi_{2}\left(V_{p p \pi}-V_{p p \sigma}\right)\left(1-\cos \phi_{2}\right)}{\left(8 \pi^{2} a_{1}^{2}\left(1-\cos \phi_{2}\right)+b^{2} \phi_{2}^{2}\right)\left(\varepsilon_{p \pi}-\varepsilon_{s}\right)\left(\varepsilon_{p \pi}-\varepsilon_{p \sigma}\right)^{3}}\right) \text {, } \\
& \rho_{2, z}^{\mathrm{A}}=\xi_{p}\left(\frac{4 \pi a_{1} b E_{\mathrm{t}} E_{z} \phi_{2}\left(V_{p p \pi}-V_{p p \sigma}\right)\left(1-\cos \phi_{2}\right)}{\left(8 \pi^{2} a_{1}^{2}\left(1-\cos \phi_{2}\right)+b^{2} \phi_{2}^{2}\right)\left(\varepsilon_{p \pi}-\varepsilon_{s}\right)\left(\varepsilon_{p \pi}-\varepsilon_{p \sigma}\right)^{2}}\right) \\
& -\xi_{p}^{2}\left(\frac{8 \pi^{2} a_{1}^{2} \sin \phi_{2}\left(1-\cos \phi_{2}\right)\left(E_{\mathrm{t}}^{2}-E_{\mathrm{n}}^{2}\right)\left(V_{p p \sigma}-V_{p p \pi}\right)+2 b^{2}\left(E_{\mathrm{t}}^{2}+E_{\mathrm{n}}^{2}\right) \sin \phi_{2} \phi_{2}^{2} V_{p p \pi}}{\left(8 \pi^{2} a_{1}^{2}\left(1-\cos \phi_{2}\right)+b^{2} \phi_{2}^{2}\right)\left(\varepsilon_{p \pi}-\varepsilon_{s}\right)\left(\varepsilon_{p \pi}-\varepsilon_{p \sigma}\right)^{3}}\right),
\end{aligned}
$$




$$
\begin{aligned}
& \rho_{2, x}^{\mathrm{M}, \mathrm{A}}=\xi_{p}\left(\frac{2 E_{\mathrm{n}} E_{z} \sin \phi_{1}\left(2 \pi^{2}\left(V_{p p \pi}+V_{p p \sigma}\right)\left(4 a_{0}^{2}-4 a_{0} a_{1} \cos \phi_{1}+a_{1}^{2}\right)+b^{2} \phi_{1}^{2} V_{p p \pi}\right)}{\left(4 \pi^{2}\left(4 a_{0}^{2}-4 a_{0} a_{1} \cos \phi_{1}+a_{1}^{2}\right)+b^{2} \phi_{1}^{2}\right)\left(\varepsilon_{p \pi}-\varepsilon_{s}\right)\left(\varepsilon_{p \pi}-\varepsilon_{p \sigma}\right)^{2}}\right. \\
& \left.+\frac{2 \pi\left(V_{p p \pi}-V_{p p \sigma}\right) b \phi_{1} E_{\mathrm{t}}\left(\left(2 a_{0}-a_{1}\right) E_{\mathrm{t}} \sin \phi_{1}+\left(2 a_{0}+a_{1}\right) E_{\mathrm{n}}\left(1-\cos \phi_{1}\right)\right)}{\left(4 \pi^{2}\left(4 a_{0}^{2}-4 a_{0} a_{1} \cos \phi_{1}+a_{1}^{2}\right)+b^{2} \phi_{1}^{2}\right)\left(\varepsilon_{p \pi}-\varepsilon_{s}\right)\left(\varepsilon_{p \pi}-\varepsilon_{p \sigma}\right)^{2}}\right) \\
& -\xi_{p}^{2}\left(\frac{4 E_{\mathrm{n}} E_{z} \sin \phi_{1}\left(2 \pi^{2}\left(V_{p p \pi}+V_{p p \sigma}\right)\left(4 a_{0}^{2}-4 a_{0} a_{1} \cos \phi_{1}+a_{1}^{2}\right)+b^{2} \phi_{1}^{2} V_{p p \pi}\right)}{\left(4 \pi^{2}\left(4 a_{0}^{2}-4 a_{0} a_{1} \cos \phi_{1}+a_{1}^{2}\right)+b^{2} \phi_{1}^{2}\right)\left(\varepsilon_{p \pi}-\varepsilon_{s}\right)\left(\varepsilon_{p \pi}-\varepsilon_{p \sigma}\right)^{3}}\right. \\
& \left.+\frac{2 \pi\left(V_{p p \pi}-V_{p p \sigma}\right)\left(2 a_{0}-a_{1}\right) b\left(E_{\mathrm{t}}^{2}+E_{\mathrm{n}}^{2}\right) \phi_{1} \sin \phi_{1}}{\left(4 \pi^{2}\left(4 a_{0}^{2}-4 a_{0} a_{1} \cos \phi_{1}+a_{1}^{2}\right)+b^{2} \phi_{1}^{2}\right)\left(\varepsilon_{p \pi}-\varepsilon_{s}\right)\left(\varepsilon_{p \pi}-\varepsilon_{p \sigma}\right)^{3}}\right), \\
& \rho_{2, x}^{\mathrm{A}, \mathrm{M}}=\rho_{2, x}^{\mathrm{M}, \mathrm{A}}-2 \xi_{p} \frac{2 \pi\left(V_{p p \pi}-V_{p p \sigma}\right) b \phi_{1} E_{\mathrm{t}}^{2}\left(2 a_{0}-a_{1}\right) \sin \phi_{1}}{\left(4 \pi^{2}\left(4 a_{0}^{2}-4 a_{0} a_{1} \cos \phi_{1}+a_{1}^{2}\right)+b^{2} \phi_{1}^{2}\right)\left(\varepsilon_{p \pi}-\varepsilon_{s}\right)\left(\varepsilon_{p \pi}-\varepsilon_{p \sigma}\right)^{2}} \\
& +2 \xi_{p}^{2} \frac{2 \pi\left(V_{p p \pi}-V_{p p \sigma}\right)\left(2 a_{0}-a_{1}\right) b\left(E_{\mathrm{t}}^{2}+E_{\mathrm{n}}^{2}\right) \phi_{1} \sin \phi_{1}}{\left(4 \pi^{2}\left(4 a_{0}^{2}-4 a_{0} a_{1} \cos \phi_{1}+a_{1}^{2}\right)+b^{2} \phi_{1}^{2}\right)\left(\varepsilon_{p \pi}-\varepsilon_{s}\right)\left(\varepsilon_{p \pi}-\varepsilon_{p \sigma}\right)^{3}}, \\
& \rho_{2, y}^{\mathrm{M}, \mathrm{A}}=\xi_{p}\left(\frac{4 \pi E_{\mathrm{t}} E_{z} \sin \phi_{1}\left(\pi\left(V_{p p \pi}+V_{p p \sigma}\right)\left(4 a_{0}^{2}-4 a_{0} a_{1} \cos \phi_{1}+a_{1}^{2}\right)+b^{2} \phi_{1}^{2} V_{p p \pi}\right)}{\left(4 \pi^{2}\left(4 a_{0}^{2}-4 a_{0} a_{1} \cos \phi_{1}+a_{1}^{2}\right)+b^{2} \phi_{1}^{2}\right)\left(\varepsilon_{p \pi}-\varepsilon_{s}\right)\left(\varepsilon_{p \pi}-\varepsilon_{p \sigma}\right)^{2}}\right. \\
& \left.-\frac{2 \pi\left(V_{p p \pi}-V_{p p \sigma}\right) b \phi_{1} E_{\mathrm{n}}\left(\left(2 a_{0}-a_{1}\right) E_{\mathrm{t}} \sin \phi_{1}+\left(2 a_{0}+a_{1}\right) E_{\mathrm{n}}\left(1-\cos \phi_{1}\right)\right)}{\left(4 \pi^{2}\left(4 a_{0}^{2}-4 a_{0} a_{1} \cos \phi_{1}+a_{1}^{2}\right)+b^{2} \phi_{1}^{2}\right)\left(\varepsilon_{p \pi}-\varepsilon_{s}\right)\left(\varepsilon_{p \pi}-\varepsilon_{p \sigma}\right)^{2}}\right) \\
& -\xi_{p}^{2}\left(\frac{E_{\mathrm{t}} E_{z} \sin \phi_{1} 4\left(2 \pi^{2}\left(V_{p p \pi}+V_{p p \sigma}\right)\left(4 a_{0}^{2}-4 a_{0} a_{1} \cos \phi_{1}+a_{1}^{2}\right)+b^{2} \phi_{1}^{2} V_{p p \pi}\right)}{\left(4 \pi^{2}\left(4 a_{0}^{2}-4 a_{0} a_{1} \cos \phi_{1}+a_{1}^{2}\right)+b^{2} \phi_{1}^{2}\right)\left(\varepsilon_{p \pi}-\varepsilon_{s}\right)\left(\varepsilon_{p \pi}-\varepsilon_{p \sigma}\right)^{3}}\right. \\
& \left.-\frac{2 \pi\left(V_{p p \pi}-V_{p p \sigma}\right)\left(2 a_{0}+a_{1}\right) b\left(E_{\mathrm{t}}^{2}+E_{\mathrm{n}}^{2}\right) \phi_{1}\left(1-\cos \phi_{1}\right)}{\left(4 \pi^{2}\left(4 a_{0}^{2}-4 a_{0} a_{1} \cos \phi_{1}+a_{1}^{2}\right)+b^{2} \phi_{1}^{2}\right)\left(\varepsilon_{p \pi}-\varepsilon_{s}\right)\left(\varepsilon_{p \pi}-\varepsilon_{p \sigma}\right)^{3}}\right)
\end{aligned}
$$

with

$$
\rho_{2, y}^{\mathrm{A}, \mathrm{M}}=\rho_{2, y}^{\mathrm{M}, \mathrm{A}}+2 \xi_{p} \frac{2 \pi\left(V_{p p \pi}-V_{p p \sigma}\right) b \phi_{1} E_{\mathrm{n}}\left(2 a_{0}-a_{1}\right) E_{\mathrm{t}} \sin \phi_{1}}{\left(4 \pi^{2}\left(4 a_{0}^{2}-4 a_{0} a_{1} \cos \phi_{1}+a_{1}^{2}\right)+b^{2} \phi_{1}^{2}\right)\left(\varepsilon_{p \pi}-\varepsilon_{s}\right)\left(\varepsilon_{p \pi}-\varepsilon_{p \sigma}\right)^{2}}
$$

and

$$
\begin{aligned}
\rho_{2, z}^{\mathrm{M}, \mathrm{A}}= & \xi_{p}\left(V_{p p \pi}-V_{p p \sigma}\right)\left(\frac{2 \pi\left(2 a_{0}+a_{1}\right) b E_{\mathrm{t}} E_{z} \phi_{1}\left(1-\cos \phi_{1}\right)-2 \pi\left(2 a_{0}-a_{1}\right) b E_{\mathrm{n}} E_{z} \phi_{1} \sin \phi_{1}}{\left(4 \pi^{2}\left(4 a_{0}^{2}-4 a_{0} a_{1} \cos \phi_{1}+a_{1}^{2}\right)+b^{2} \phi_{1}^{2}\right)\left(\varepsilon_{p \pi}-\varepsilon_{s}\right)\left(\varepsilon_{p \pi}-\varepsilon_{p \sigma}\right)^{2}}\right) \\
& -\xi_{p}^{2}\left(2\left(E_{\mathrm{t}}^{2}+E_{\mathrm{n}}^{2}\right) \sin \phi_{1} \frac{2 \pi^{2}\left(V_{p p \pi}+V_{p p \sigma}\right)\left(\left(4 a_{0}^{2}+a_{1}^{2}\right)-2 a_{0} a_{1} \cos \phi_{1}\right)+b^{2} \phi_{1}^{2} V_{p p \pi}}{\left(4 \pi^{2}\left(4 a_{0}^{2}-4 a_{0} a_{1} \cos \phi_{1}+a_{1}^{2}\right)+b^{2} \phi_{1}^{2}\right)\left(\varepsilon_{p \pi}-\varepsilon_{s}\right)\left(\varepsilon_{p \pi}-\varepsilon_{p \sigma}\right)^{3}}\right)
\end{aligned}
$$


with

$$
\rho_{2, z}^{\mathrm{A}, \mathrm{M}}=\rho_{2, z}^{\mathrm{M}, \mathrm{A}}+2 \xi_{p} \frac{2 \pi\left(V_{p p \pi}-V_{p p \sigma}\right)\left(2 a_{0}-a_{1}\right) b E_{\mathrm{n}} E_{z} \phi_{1} \sin \phi_{1}}{\left(4 \pi^{2}\left(4 a_{0}^{2}-4 a_{0} a_{1} \cos \phi_{1}+a_{1}^{2}\right)+b^{2} \phi_{1}^{2}\right)\left(\varepsilon_{p \pi}-\varepsilon_{s}\right)\left(\varepsilon_{p \pi}-\varepsilon_{p \sigma}\right)^{2}} .
$$

\section{References}

(1) Pastawski, H. M.; Medina, E. 'Tight binding' methods in quantum transport through molecules and small devices: From the coherent to the decoherent description. Rev. Mex. Fis. 2001, 47, 1-23.

(2) Varela, S.; Mujica, V.; Medina, E. Effective spin-orbit couplings in an analytical tightbinding model of DNA: Spin filtering and chiral spin transport. Phys. Rev. B 2016, 93, 155436.

(3) Hubac, I.; Wilson, S. Brillouin-Wigner Methods for Many-Body Systems; Progress in Theoretical Chemistry and Physics; Springer Netherlands: Dordrecht, 2010; Vol. 21. 